\title{
Visual response augmentation in cat (and macaque) LGN: potentiation by corticofugally mediated gain control in the temporal domain
}

\author{
Javier Cudeiro, Casto Rivadulla and Kenneth L. Grieve
}

\begin{abstract}
Visual responses of neurons are dependent on the context of a stimulus, not only in spatial terms but also temporally, although evidence for temporally separate visual infuences is meagre, based mainly on studies in the higher cortex. Here we demonstrate temporally induced elevation of visual responsiveness in cells in the lateral geniculate nucleus (LGN) of cat and monkey following a period of high intensity (elevated contrast) stimulation. This augmentation is seen in $40-70 \%$ (monkey-cat) of cells tested and of all subtypes. Peaking at $\sim 3$ minfollowing the period of intense stimulation, it can last for $10 \pm 12$ minand can be repeated and summed in time. Furthermore, it is dependent on corticofugal input, is seen even when high contrast stimuli of orthogonal orientation are used and therefore results from a/any prior increase in activity in the retino-geniculo-striate pathway. We suggest that this reflects a general mechanism for control of visual responsiveness; both a flexible and dynamic means of changing effectiveness of thalamic activity as visual input changes, but also a mechanism which is an emergent property of the thalamo-corticothalamic loop.
\end{abstract}

Keywords: context dependent, contrast mediated, cortico-geniculate, response enhancement, thalamo-corticothalamic loop

\section{Introduction}

In the past few years there have been many findings implicating the dorsal lateral geniculate nucleus (LGN) in the processing of visual information en route to the visual cortex, rather than the more traditional view of this nucleus - devoted to the faithful transmission of such information, without alteration. This visual processing has involved alteration, e.g. the strength of receptive field surround (Sillito \& Kemp, 1983), the degree of orientation bias (Vidyasagar, 1984) or the addition of influences from the non-dominant eye (Pape \& Eysel, 1986; Murphy \& Sillito, 1987).We can regard all such receptive field modifications as purely spatial, i.e. dealing with the spatial influences on on-going visual responses, either within or outside the classical receptive field (Maffei \& Fiorentini, 1976; Nelson \& Frost, 1978; Gilbert \& Wiesel, 1991; DeAngelis et al, 1992; Sillito et al., 1995; Levitt \& Lund, 1997). Yet in a visual system designed to work continuously and seamlessly during prolonged observation of the visual world, temporal changes in visual responses should also be considered (Logothetis et al., 1996). The temporal scale is a variable much less studied in visual neurophysiology (not so in psychophysics).Here, by temporal scale we mean not only the evolution in time of visual responses but, as importantly, the effect of the visual image preceding the scene currently under analysis. Traditional neurophysiological studies almost exclusively use repetitive, identical visual stimuli in order to average out variability due to 'noise', thereby ignoring or removing any such effects. One of the simplest temporal changes which could be evoked would be a modulation of gain. It is well known that neuronal responses to a given stimulus may be altered by the prior exposure of the cell under test to repetitive stimulation, e.g. using trains of electrical pulses to give rise to the well-known phenomena of short- and long-term potentiation (STP and LTP). This has been particularly well studied in the hippocampus, but is also documented in the visual cortex (V1) of both developing and adult animals (Bliss \& Gardner-Medwin, 1973; Bliss \& Lomo, 1973; Artola \& Singer, 1987; Bliss \& Collingridge, 1993; Kirkwood \& Bear, 1994; Fox, 1995).In an analogous fashion, we have recently shown that an iontophoretic application of excitatory amino acids to LGN cells could induce enhanced excitability which extended in time beyond the period of application, while a similar period of chemically induced excitation by application of 
acetylcholine was without effect (Rivadulla et al., 1998). The LGN receives synaptic input from many sources, but the major pathways utilizing excitatory amino acid receptors are from the retina and visual cortex. Here we have sought to identify the source of this change in excitability, and further characterize its properties, using traditional visual stimulus paradigms but in such a way as to look to changes in time of the responses to such stimuli.

\section{Materials and methods}

Experiments were carried out on adult cats. Animals were anaesthetized with halothane $(0.1-5 \%)$ in nitrous oxide $(70 \%)$ and oxygen $(30 \%)$, and paralysed with gallamine triethiodide $(10 \mathrm{mg} / \mathrm{kg} / \mathrm{h})$.EEG, ECG, expired $\mathrm{CO} 2$ and temperature were monitored and maintained continuously, adjusting anaesthetic levels to maintain a state of light anaesthesia (Cudeiro et al., 1997). Single units were recorded extracellularly from the LGN using multibarrelled glass micropipettes or tungsten in glass microelectrodes, and recordings in the visual cortex were made with tungsten in glass electrodes. Single unit data were collected and visual stimuli produced under computer control. We investigated using two basic paradigms: firstly, we established control responses to a drifting grating of medium contrast (0.20.6 , mean luminance of $13 \mathrm{~cd} / \mathrm{m}^{2}$ ); secondly, we electrically stimulated the optic chiasm at $20 \mathrm{~Hz}$ (bipolar electrodes across the optic chiasm, monophasic pulses $0.05 \mathrm{~ms}$ duration, up to $1 \mathrm{~mA}$ ) with or without visual stimulation using a stimulus of similar properties to the test stimulus; finally retest the grating response at 1, 3, 6 and 12 min following cessation. Both control and test stimuli were presented for a period of $\sim 1 \mathrm{~min}$ [18 x 4s stimulus periods (electrical and/or visual, total $72 \mathrm{~s}$ ) separated by $1 \mathrm{~s}$ intervals] and responses averaged into peri-stimulus time histograms (PSTHs).Secondly, we also established control responses to a drifting grating, but then visually stimulated with a high-contrast non-saturating stimulus (spatial and temporal frequency and direction as for the test stimulus, contrast 0.8-0.9, orientation the same as or orthogonal to the test, see Results), retesting also at 1, 3,6 and 12 min. Again, each stimulus was presented as outlined above. Average spike counts for the response to 1 cycle of the grating were calculated and results were compared statistically using the Wilcoxon matched pairs test. Differences between control and test giving rise to $P<0.05$ were considered significant. Maximum and minimum firing rates during visual stimulation were also compared, as were periods of spontaneous activity in the absence of stimulation. Visual stimuli were presented monocularly to the dominant eye via a computer monitor (SONY Multiscan GDM-17”, $80 \mathrm{~Hz}$ refresh rate), under computer control. In three cats, areas 17 and 18 of the visual cortex on one side were removed by aspiration; histological analysis confirmed that the lesions extended well beyond those regions of areas 17 and 18 that would have been activated by our stimuli. All recordings are from cells with receptive field eccentricities within $12^{\circ}$ of the area centralis. A further recording experiment was carried out on one adult monkey (Macaca mulatta), in which the methods were similar, except that in this case isoflurane was the anaesthetic agent of choice. All experiments complied with the guidelines of the Spanish Physiology Society, the International Council for Laboratory Animal Science and the European Union (86/809).

\section{Results}

Electrical stimulation of the retino-geniculate pathway produces response augmentation in a small proportion of LGN cells

We tested the effect of electrical stimulation (see Materials and methods) of the optic chiasm, during the absence of visual stimulation, on 13 LGN cells. In a small proportion, some two out of 13 (15\%), we observed a transient increase in visual responsiveness following the period of stimulation. One of these is illustrated in Fig.1. Here we show: (i) the individual sweeps of the grating (10 trials each comprising 8 grating cycles) in the control situation and at $3 \mathrm{~min}$ following the stimulation; (ii) the combined PSTHs and (iii) examples of the actual spike waveforms taken from the spontaneous discharge. The visual responses were clearly elevated following the period of stimulation. Note that spontaneous discharge was also elevated (this issue is addressed further below). A second example is shown in Fig.2. In this case we show the temporal characteristics of the augmentation effect. These PSTHs show the 8 cycles in each sweep combined and the 10 sweeps averaged. There was increased visual response 1, 3 and 6 min following the electrical stimulation, returning to prestimulation control levels by $12 \mathrm{~min}$. Interestingly, the effect seen was both qualitatively and quantitatively similar to our findings with iontophoretic application of excitatory amino acids to cells of the LGN (Rivadulla et al., 1998). 


\section{After 3' of electrical}

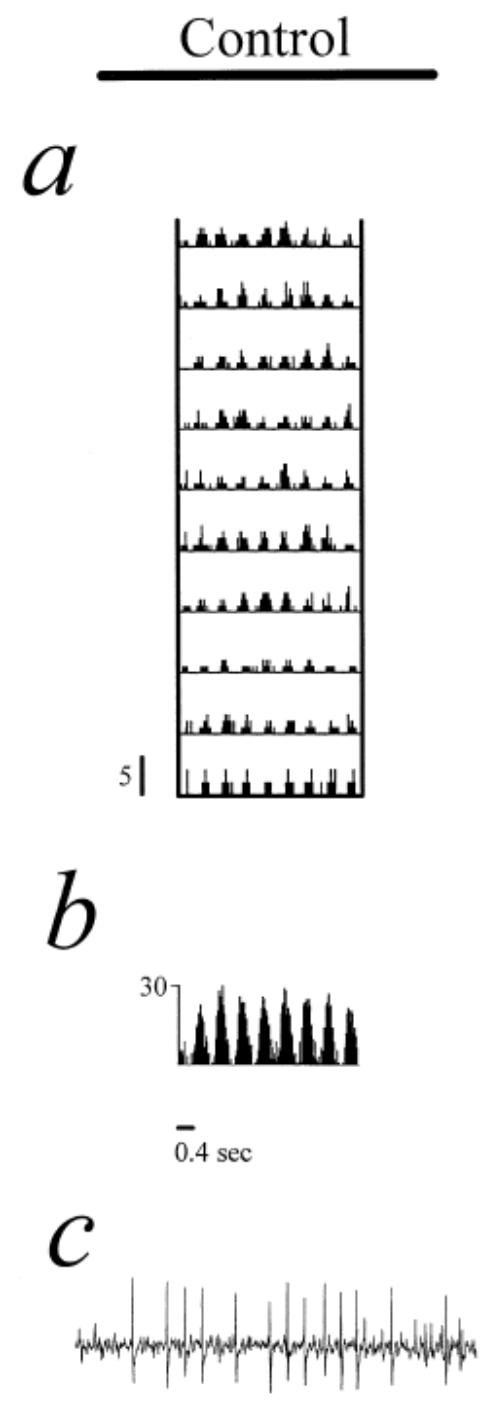

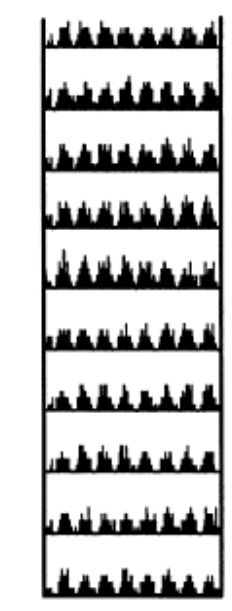

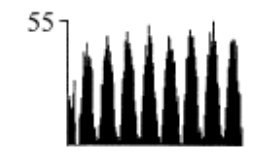

FIG.1.The effect of electrical stimulation of the optic chiasm on the visual responsiveness of a single LGN cell.(a) The left side of the figure shows PSTHs of individual trials of the presentation of the test grating prior to electrical stimulation. Each trial comprises 8 cycles of a drifting grating. The right side shows similar PSTHs taken 3 min after the end of a 1-min electrical stimulation paradigm (see Materials and methods, bin size $25 \mathrm{~ms}$, vertical scale in spikes per bin).(b) The responses in (a) averaged together to show the response over a single cycle of the drifting grating.(c) Representative segments spike waveforms during spontaneous activity.Analogue signals were digitized at a rate of $10 \mathrm{kHz}$ and stored on computer 


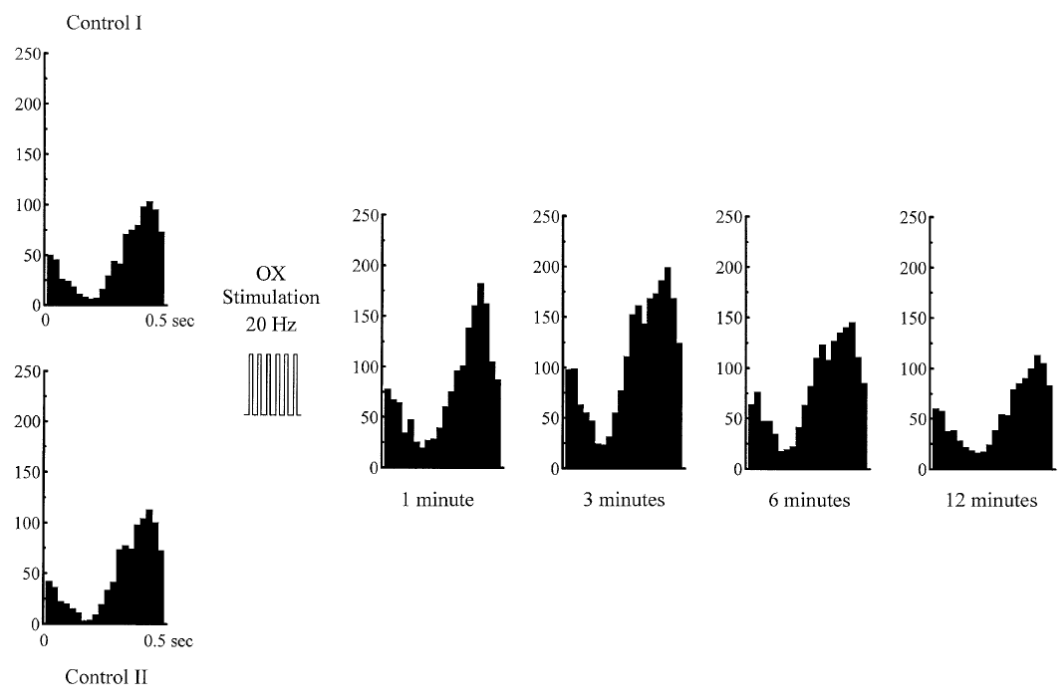

FIG .2. The effect of electrical stimulation of the optic chiasm on the visual responsiveness of a single LGN ON X cell. Repeated control responses are shown in the left-most two PSTHs, followed by responses after electrical stimulation of the optic chiasm. Responses were measured 1, 3, 6 and 12 min after termination of the electrical stimulation. Bin size, $25 \mathrm{~ms}$.

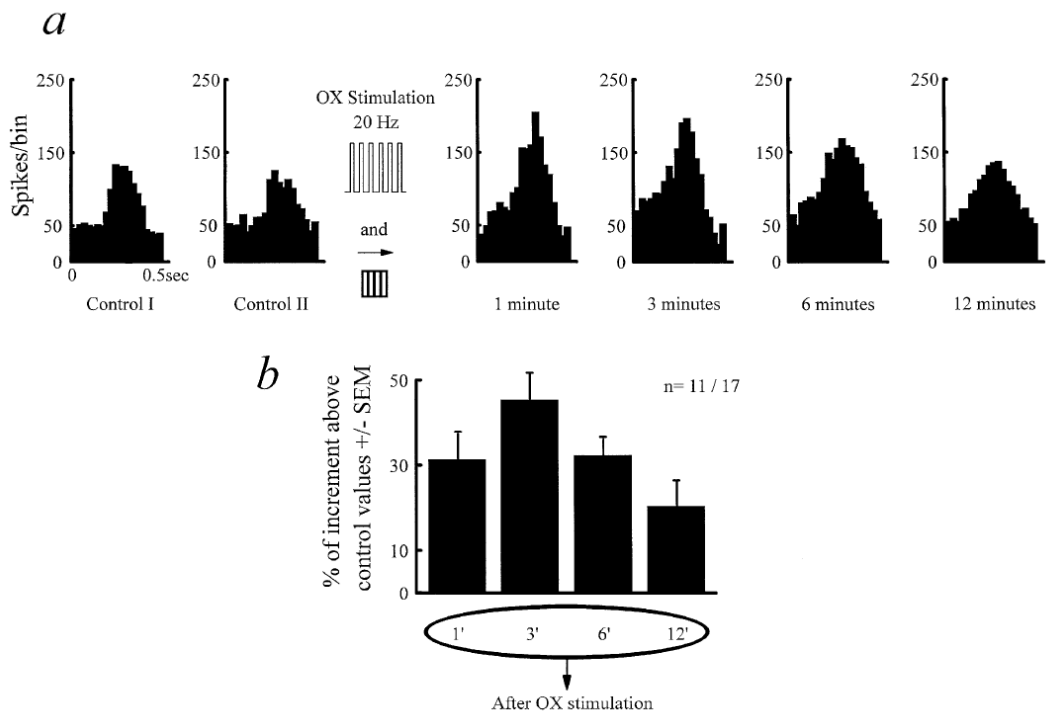

FIG .3. The effect of combined electrical stimulation of the optic chiasm with visual stimulation on the visual responsiveness of a single LGN ON Y cell.(a) Repeated control responses are shown in the left-most two PSTHs, followed by responses after electrical stimulation of the optic chiasm $(20 \mathrm{~Hz})$ with a drifting sinusoidal grating visual stimulus $\left(0.5 \mathrm{cycle} /{ }^{\circ}, 2 \mathrm{cycles} / \mathrm{s}\right.$, contrast 0.4$)$. Responses were measured 1, 3, 6 and 12 min after termination of the stimulation period. Bin size, 25 ms.(b) Histogram of the averaged responses for the population of cells showing statistically significant potentiation. 

alone

We reasoned that a time-locked electrical stimulation $(20 \mathrm{~Hz})$ was non-physiological (essentially firing all retinal efferents simultaneously), and decided to test the combination of electrical with visual stimulation, thereby combining "natural" stimulation with a general heightened excitation. Controls providing $\sim 1 \mathrm{~min}$ of visual stimulation using the drifting grating appropriate for the cell under test showed no response augmentation on any cell tested. We next tested the combination of visual and electrical stimulation. We were interested to find that the proportion of cells showing potentiation was markedly increased (11 out of 17 cells, 65\%).An example is shown in Fig.3. Here again the responses to a standard visual stimulus (see controls) were elevated immediately following the combined visual and electrical stimulation, remaining elevated for several minutes, before returning to control levels at $\sim 12-18$ min following stimulation. In these 11 cells, responses compared with controls were increased on average by $31 \pm 6$ at $1 \mathrm{~min}, 45 \pm 6$ at $3 \mathrm{~min}, 32 \pm 6$ at $6 \mathrm{~min}$ and $20 \pm 6$ at $12 \mathrm{~min}$.

The effectiveness of paired stimulation is further illustrated in Fig.4. In this cell we studied visual and electrical stimulation alone, and a combination of the two using three stimulus durations, 16,32 and $72 \mathrm{~s}$, compared with the full length of visual or electrical stimulation alone. In each case the effect on the cell was re-tested by using the control visual stimulus 3 min after the "potentiating" stimulation ceased, and the magnitudes of these responses are illustrated. Here, then, there was a clear effect of the paired stimulation with no obvious change in response following either visual or electrical stimulation alone, which showed not only a potentiated response following the paired stimulation, but also revealed a timedependent modulation.

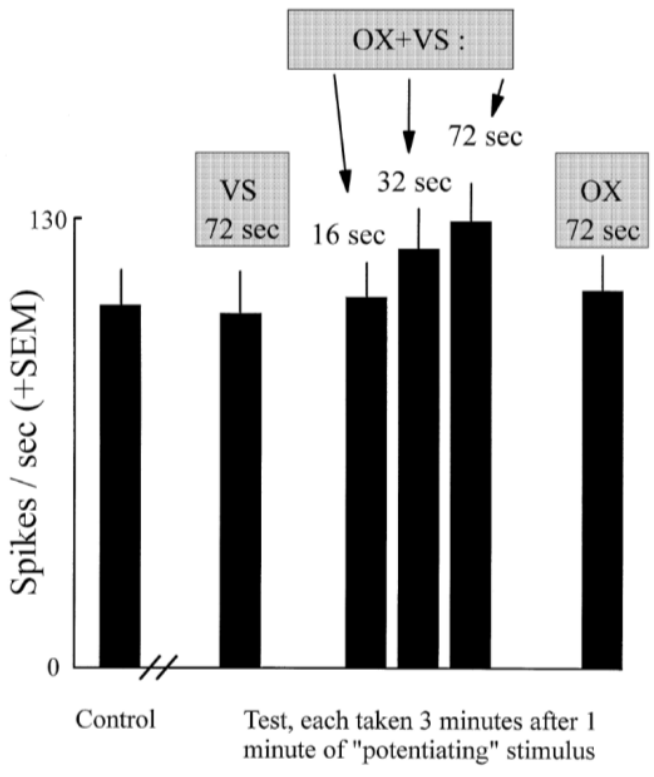

FIG .4. Bar diagram showing the dependence of the potentiation obtained with electrical stimulation upon the simultaneous presence of visual stimulation. Bars show the mean cell discharge $3 \mathrm{~min}$ after the electrical or visual stimulation (alone or paired).OX, optic chiasm electrical stimulation; VS, visual stimulation (sinusoidal drifting grating with optimal characteristics to activate the cell).Left $=$ control, then from left to right, full duration vision alone, followed by paired stimulation for a duration of 16,32 and $72 \mathrm{~s}$, finally electrical alone for the full duration.

\section{Augmentation is obtained by contrast manipulation alone}

If heightened excitation plus visual stimulation was more effective than either alone, another route by which to achieve the same end might be to alter the visual stimulus, e.g. by increasing contrast. We tested relatively prolonged, continuous visual stimulation with a high-contrast version of the test stimulus. All other attributes of the stimulus were kept the same as the test case. As illustrated by the single unit responses in Fig.5, this paradigm was also capable of inducing a response augmentation following the stimulation period, which similarly peaked at $\sim 3$ and $6 \mathrm{~min}$, and lasted between 6 and $12 \mathrm{~min}$. The 
response of the cell during the visual stimulation period is also shown (enlarged PSTH) - the response magnitude is significantly increased during the presentation (note scale bars) but is clearly non-saturating. Notably, however, this type of augmenting effect was seen in 24 out of 35 (68\%) of cells tested. A second example is shown in expanded form in Fig.6. Here again there is evidence of elevated spontaneous discharge as well as augmented visual responses. In these 24 cells, responses compared with controls were increased on average by $38 \pm 6$ at $1 \mathrm{~min}, 60 \pm 6$ at $3 \mathrm{~min}, 51 \pm 6$ at $6 \mathrm{~min}$ and $11 \pm 6$ at $12 \mathrm{~min}$. Response augmentation was also seen when stimulation was reduced to $32 \mathrm{~s}$ duration, was much less with $16 \mathrm{~s}$ and was not observable with only $10 \mathrm{~s}$ of stimulation (data not shown).In most cases the gratings, test and stimulating, subtended $32 \times 24^{\circ}$ of the visual angle, greatly exceeding the dimensions of the receptive field of the cell under test. However, smaller grating patches of $10 \times 10^{\circ}$ were also effective. Importantly, in two out of three cells tested, augmentation was seen when the patch size was restricted to the area defined as the classical receptive field. Cells showing augmentation were found in the same animal and same penetrations with cells which did not show augmentation. There was no obvious correlate in terms of cell type (ON/OFF and X/Y), depth within the LGN, etc.by which we could separate the two groups.

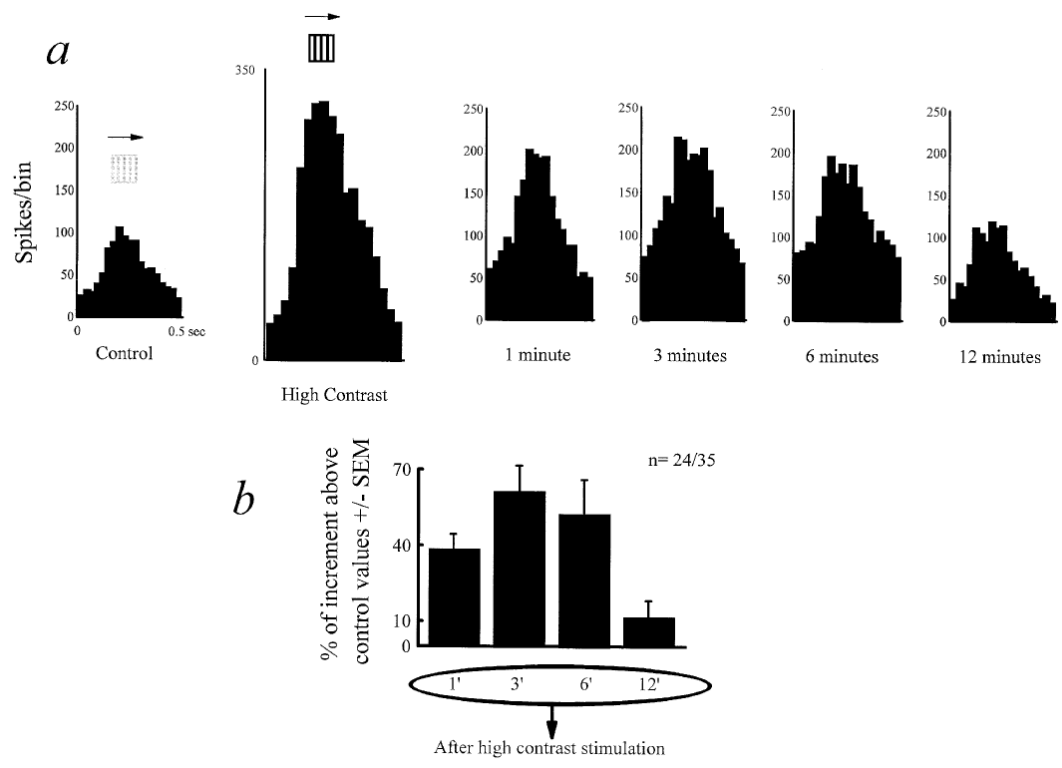

FIG .5. The effect of high-contrast visual stimulation on subsequent visual responses. (a) Left, control PSTH (contrast 0.2 ) obtained prior to visual stimulation with a stimulus of high contrast (0.9), but otherwise identical; note the elevated (see scale bars) but otherwise similar visual responses during high-contrast stimulation. Further responses were then measured 1, 3, 6 and 12 min after the end of the high-contrast stimulation. Note the increased response magnitudes and the similarity with the electrical/combined electrical standard contrast visual effects illustrated in Figs 2 and 3.In this case, the cell under test was a ON X cell. Bin size, 25 ms. (b) Population histogram for cells tested with the same paradigm. The peak of the response augmentation was seen at $3 \pm 6$ min after high-contrast stimulation, and baseline levels were reached in most cases by $12 \mathrm{~min}$

In the examples shown in Figs 1 and 6, there was an obvious increase in spontaneous activity following high-intensity stimulation in each case. This is more graphically illustrated in Fig.7.The cell originally shown in Fig.6 (now Fig.7a) has a relatively low spontaneous activity and evoked response; here the elevated response to the grating stimulus may simply be the result of a general increase in firing as seen in the basal discharge. However, in Fig.7b, the cell (from Fig.1) shows a much higher degree of augmentation for the visually driven responses than the activity in the absence of visual stimulation. While there would also appear to be a direct relationship between absolute firing rate and the degree or potency of the augmentation effect, this did not hold for the population as a whole. On average, background activity for the cells showing augmentation following high-contrast stimulation was elevated by $33 \%$, compared with the evoked discharge augmentation of $60 \%$. 


\section{Control}

$a$

5

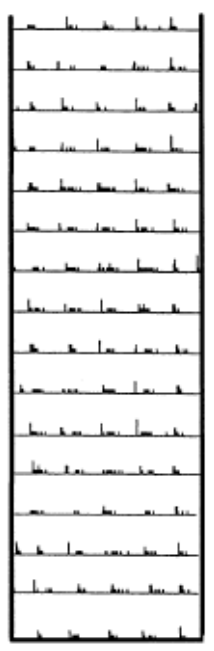

$b$

"limil

$\overline{0.8} \mathrm{sec}$

$C$

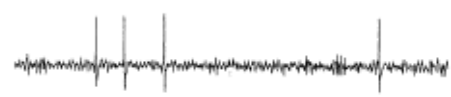

After 3' of high

contrast stimulation

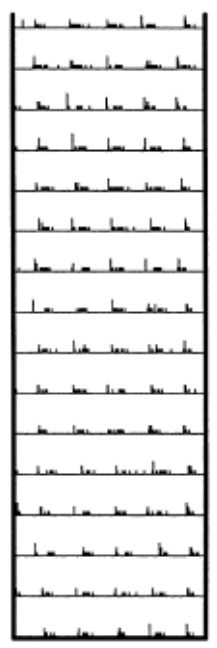

"Wilik

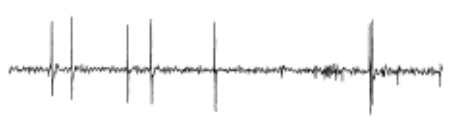

$100 \mathrm{msec}$

FIG .6. The effect of high-contrast visual stimulation on the visual responsiveness of a single LGN cell.(a) The left side of the figure shows PSTHs of individual trials of the presentation of the test grating prior to high-contrast stimulation. Each trial comprises 5 cycles of a drifting grating. The right side of (a) shows similar PSTHs taken 3 min after the end of a 1-min high-contrast stimulation (bin size, $25 \mathrm{~ms}$, vertical scale in spikes per bin). (b) The responses from (a) averaged together, as in Fig.1. (c) Representative segments spike waveforms during spontaneous activity. 

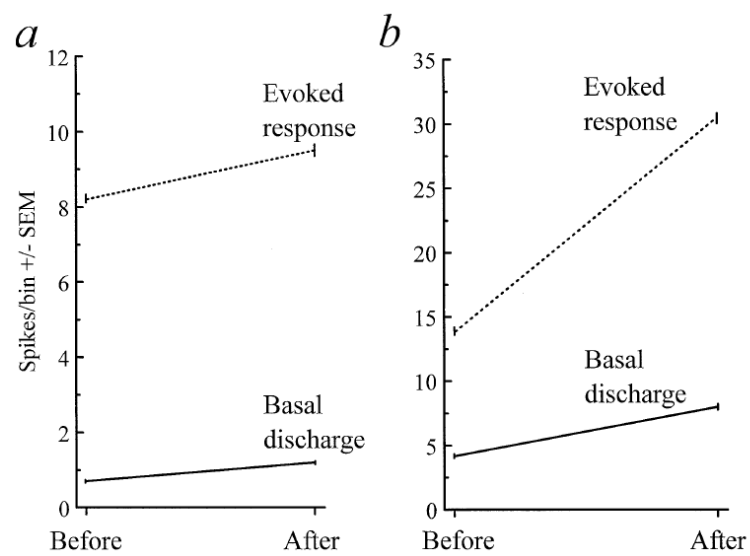

FIG .7. The effect of high-intensity stimulation on background activity and evoked responses.(a) Responses of the cell illustrated in Fig.6, both basal discharge and evoked responses are essentially equally elevated by high-contrast stimulation.(b) Responses of the cell illustrated in Fig.1.The augmentation is most evident in the electrically evoked responses; background activity is much less affected.

\section{Augmentation may be corticofugally mediated}

Because previous pharmacological data from our own laboratory (Rivadulla et al., 1998) have also suggested that this type of augmentation is not directly related to modulatory inputs to the LGN, e.g. the cholinergic input from the brainstem, we explored the possibility of a cortically mediated influence. Recordings were obtained from LGN cells after the corticofugal input was surgically removed in two different conditions: from the outset of the experiment; or in the middle of an electrode penetration after several neurons were recorded under normal conditions. In both cases, the results taken after decortication were not significantly different and have been pooled. Removal of the visual cortex resulted in a major, near complete, loss of the observed, high-contrast induced potentiation. These observations are illustrated in Fig.8a.This compares the responses of two on-centre X cells recorded in the same experiment, one before, and one after the cortex was removed. The cell receiving intact corticofugal feedback shows a clear augmented response $3 \mathrm{~min}$ after high-contrast stimulation, in comparison with prior control levels, while responses of the cell recorded after decortication were completely unchanged. Figure 8b summarized these data from the population of cells studied either with or without cortical input. There is an obvious and highly significant reduction in the percentage of cells showing augmentation in the absence of corticofugal input (63\% versus 6\%).In another experiment, when a similar stimulus protocol was applied to a population of nine histologically confirmed cortical layer 6 cells (source of cortico-fugal feedback to the dLGN) no augmentation was seen in any case, although the cells were clearly well driven during the high-contrast stimulation (middle PSTH), showing neither adaptation nor fatigue to this prolonged high-intensity stimulation. Responses only equal to control levels were seen at all times after high-intensity stimulation. These cortical responses are illustrated by the example shown in Fig.8c. In Fig.9, we show data obtained from LGN cells in a typical decortication experiment. Here, nine cells were recorded before decortication, and 17 cells after. For a typical cell before decortication and a second cell after, responses prior to and $3 \mathrm{~min}$ after high-intensity stimulation are shown as histograms. There was clear and significant enhancement in both basal discharge and visually elicited responses prior to decortication, and no significant differences after. 
$a$

With visual cortex $\quad$ Without visual cortex

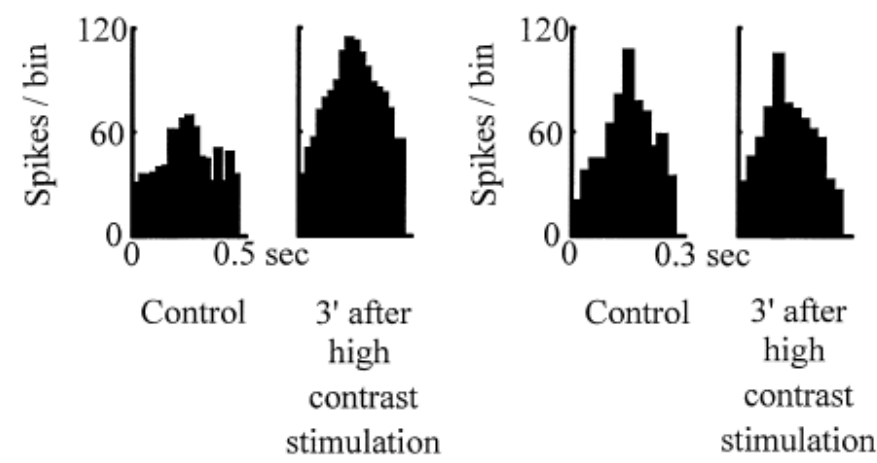

$b$

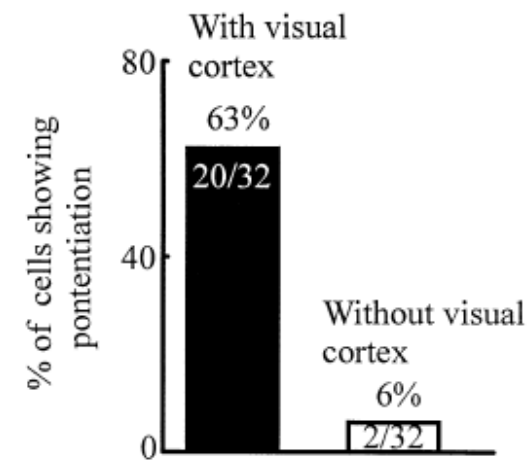

C

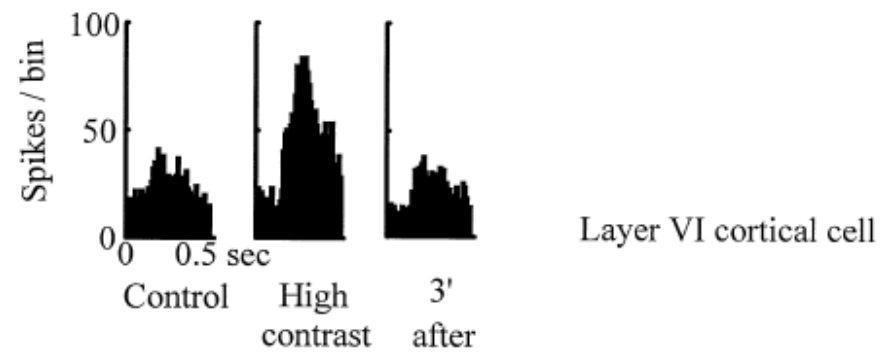

FIG .8. The contribution of corticofugal feedback to the augmentation of visual responses I.(a) Data refer to two on-centre X cells recorded during the same experiment prior to (left) and after (right) decortication. In both cases the PSTHs show the cell control and test responses $3 \mathrm{~min}$ after stimulation with a high-contrast grating (details as in Fig.2). (b) Summary histogram of the results obtained for the population of cells studied with and without corticofugal input.(c) PSTHs documenting the responses of a typical cortical layer VI cell being tested with a high-contrast stimulus. The average change in response magnitude following high-contrast stimulation for the population was $\sim 3 \%$ and was not significantly different to control values. 

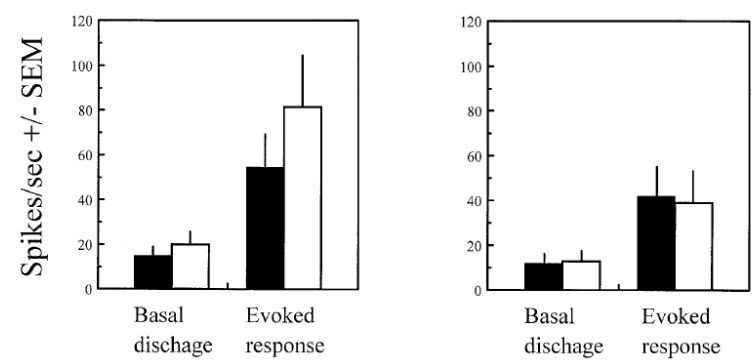

Control

After 3' of stimulation

FIG 9. The contribution of corticofugal feedback to the augmentation of visual responses in the LGN II. Results from one complete experiment, in which nine control cells were recorded prior to decortication and 17 cells recorded following aspiration of V1.Histograms show the mean spontaneous activity and mean visual responses prior to and after high-intensity stimulation, \pm SEM in cells before decortication (left, $n=9$ ) and after decortication (right, $n=17$ )

\section{Augmentation is orientation independent}

Seven cells were tested with a high-contrast full-field grating at $90^{\circ}$ to the test stimulus. Of these cells, three showed augmentation which was not significantly different to the responses produced by a grating iso-orientated to the high-contrast stimulus. This is illustrated in Fig.10. Although there appears to be a systematic decrease in the responses to the $90^{\circ}$ grating, this was not statistically significant.

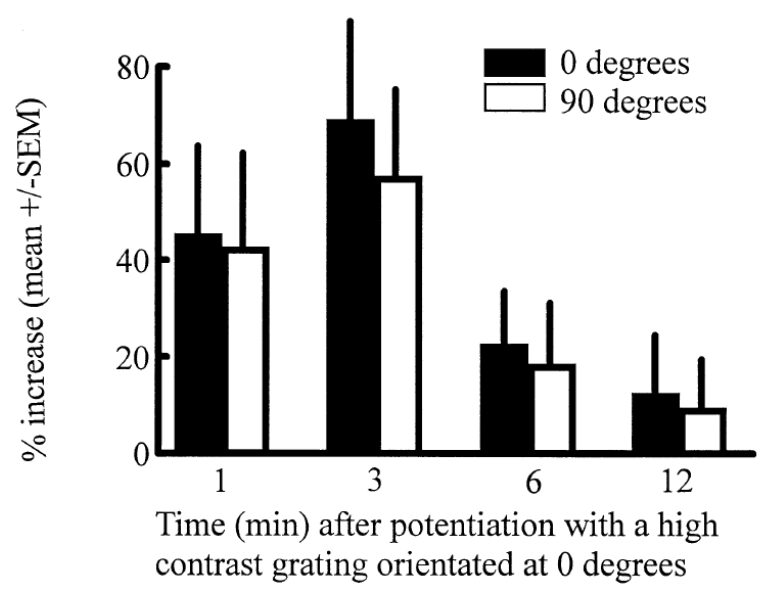

FIG .10. The effect of stimulus orientation on augmentation. The bar histogram shows the average response of LGN cells showing augmentation following a high-contrast stimulus which was $90^{\circ}$ to the test orientation. Following stimulation, the cells were tested with randomly interleaved gratings of 0 and $90^{\circ}$.Augmentation was clearly seen and was not statistically significantly different in the two stimulus conditions.

\section{Similar results were obtained in monkey}

In one monkey, high-contrast visual stimulation resulted in response augmentation in 11 out of 30 cells (nine out of 25 parvo-cellular and two out of five magno-cellular).Although this is a smaller proportion than was seen in the cat, the sample size precludes further detailed interpretation. The basic phenomenon is illustrated in Fig.11. Here we show a cell showing the type of potentiation we have previously illustrated in cat LGN. In this case a more detailed analysis also showed a response which is readily reproducible (Fig.11a) - a second high-contrast stimulus period was followed by a near identical pattern of response potentiation. Interestingly, in this, and one other cell in the monkey (and, importantly, 
two also in the cat), we re-stimulated during the period of potentiation and saw clear summation (Fig.11b). In this particular case a third period of stimulation was also effective, but only for $1 \mathrm{~min}$, after which responses could not be further augmented and slowly declined in magnitude to control levels over the subsequent $18 \mathrm{~min}$. In these 11 cells from the macaque, responses compared with controls were increased on average by $45 \pm 6$ at $1 \mathrm{~min}, 53 \pm 6$ at $3 \mathrm{~min}, 26 \pm 6$ at $6 \mathrm{~min}$ and $20 \pm 6$ at $12 \mathrm{~min}$.
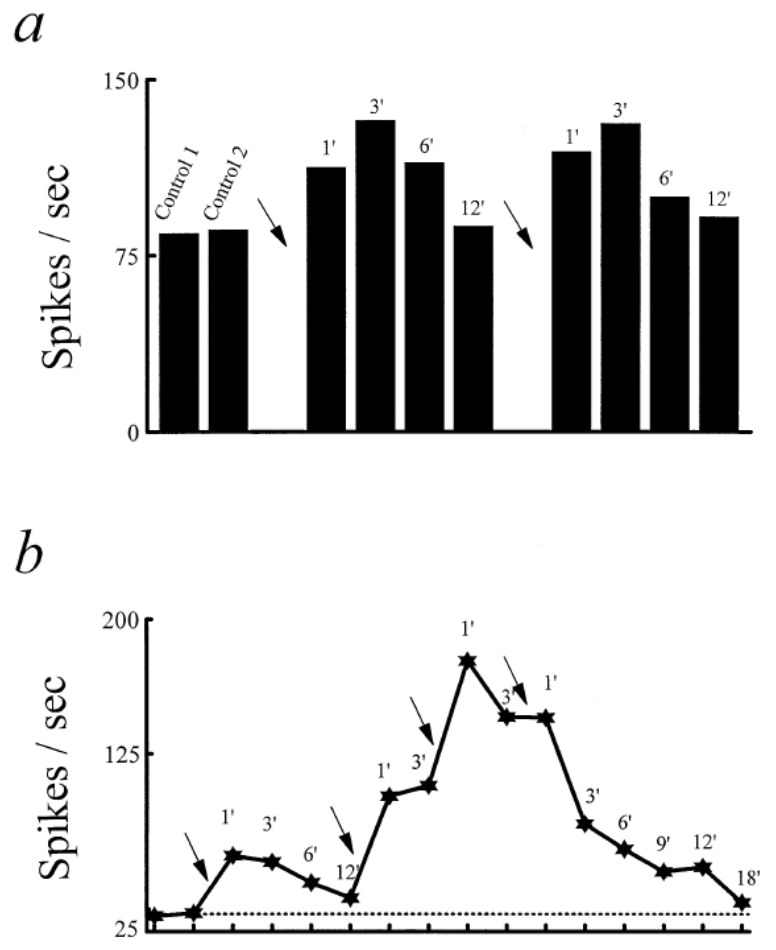

FIG .11. Data obtained from monkey LGN. (a) Single cell data from a magno-cell in the monkey LGN. The augmentation effect induced by exposure to $1 \mathrm{~min}$ of a high-contrast drifting grating was clearly reproducible - a second period of stimulation following recovery from the first is shown as a histogram.(b) A second monkey LGN cell - in this case the cell (parvo-cellular) was stimulated four times in total (arrows) showing a measurable degree of summation. Dashed line represents control response levels.

\section{Discussion}

From the data we have presented above, we have derived a very simple hypothesis; the "instantaneous" firing rate of many geniculate cells is related to the intensity of their firing up to several minutes prior to any point of measurement. Basically, a period of heightened, driven activity (in our case $1 \mathrm{~min}$ ) results in higher background and, particularly, visually evoked firing, which extends in time by several minutes beyond the period of heightened activity. This activity seems to be a corticofugally mediated effect, but is not orientation specific. Although corticofugally mediated, the effect originates within the LGN, as it is not seen in cortical cells providing the input to LGN, but, we predict, should be seen in cortical cells receiving this augmented geniculate signal, presumably at least within layer IV (although this aspect remains to be tested).We propose that we are describing a mechanism for a general temporal gain control of geniculate responsivity driven by corticofugal activation, possibly via metabotropic receptors on LGN cells, acting as 'modulators' of the transfer of information from retina to cortex (Sherman \& Guillery, 1998).In fact, however, there are two obvious competing theories to provide an explanation of the data we present here. Firstly, it can be suggested that the continued, repetitive stimulation of the visual field merely resulted in the temporary fatigue of inhibitory interneurons within the LGN driven by direct cortico-geniculate input.Corticofugal input is known to enhance intrageniculate inhibition as, e.g. seen when stimuli such as bars of different length are used (enhanced end-inhibition as a result of cortical input, Murphy \& Sillito, 1987). This inhibition is extended spatially in retinotopic terms and would be compatible with the $32 \times 24^{\circ}$ visual stimulus we used routinely, and with the optic chiasm electrical stimulation, which is likely to activate much of the axonal output of the retina. However, 
it is less compatible with the effectiveness of smaller $10 \times 10^{\circ}$ patches, or those covering only the receptive field of the cell under test, as in their study, Murphy \& Sillito (1987) required lesion-extended regions of both cortical areas 17 and 18 to achieve disinhibition within the LGN.A second hypothesis suggests that this augmentation is the result of enhanced excitability of LGN cells, following activation of a direct excitatory cortico-fugal input. Here the extended duration of the augmentation beyond the period of intense stimulation could be due to activation of a separate subset of excitatory amino acid receptors (metabotropic) known to be present on LGN cells, and activated by cortico-fugal input (McCormick \& von Krosigk, 1992).Indeed, O'Connor and colleagues (O'Connor et al., 1994) have shown in the rat hippocampus that one form of LTP of N-methyl-D-aspartate (NMDA) responses required activation of metabotropic glutamate receptors. Thus, our data provide the interesting proposition that the descending cortico-fugal influence uses a metabotropic receptor activation to "kick start" a short duration NMDAmediated potentiation - the metabotropic activation requiring a high level of activity in the corticofugal line. However, an arbitrary choice between the disinhibition and excitation models we have proposed may in fact be a misleading one, as in reality elements of both mechanisms could combine to produce the observed effects. These are, however, simplistic ideas, given the complexity now known to exist at the level of the LGN and in its relationship with the cortex. Accumulating recent evidence suggests that retinal input may also access metabotropic receptors within the LGN, activation of which has a time course commensurate with the effects we have observed (McCormick \& von Krosigk, 1992).However, these receptors are of the mGluR5 subtype, while those associated with cortico-geniculate feedback are of the mGluR1 subtype. These retinally associated receptors appear to involve synaptic triads and facilitate feedforward inhibition (Godwin et al., 1996a; Cox et al., 1998), and are therefore unlikely to be involved in the effects we have observed. Another hypothesis regarding the role of corticofugal feedback onto LGN metabotropic receptors suggests that such feedback shifts the response 'mode' of LGN cells from burst to tonic firing, thereby enhancing the information throughput of the retino-geniculo-cortical circuit (Godwin et al., 1996b). However, we did not consistently observe such bursting characteristics, either before or after stimulation, and the high efficacy of information transfer in burst versus tonic mode is consistent with the view that burst and tonic modes may both occur in states of light anaesthesia such as we describe here (Guido et al., 1995; Sherman, 1996; Reinagel et al., 1999). It remains possible that this reflects differences in anaesthetic protocols.

A globally arousing stimulus may well accentuate the visual responsiveness of the LGN and account for the data we have presented. While we would accept such a hypothesis based only on the evidence of our electrical stimulation of the optic nerve, or perhaps even on full-field high-contrast visual stimulation, it is more difficult to believe when high-contrast stimulation was restricted in size. Paradoxically, however, it is indeed a form of arousal we believe to be the mechanism at work. On one level, the effects we have observed here are very similar to those found using electrical stimulation of the parabrachium (Uhlrich et al., 1995), with increases both in background and visually evoked activities. In that case, however, enhanced responses were seen essentially only during stimulation, there was no further increase after stimulation such as we have observed here. This difference and the lack of enhancement following decortication suggest that this is not the mechanism involved in our observations.

Indeed, it is interesting to note that, on first glance, not all of our LGN cells appeared to be affected, and we could find no defining characteristic which would predict whether or not a cell would show augmentation. However, these experiments necessarily involved work on an anaesthetized animal, and it remains possible, even likely, that the effects we observed could be far greater in a conscious awake, behaving preparation. Also, we opted for a robust statistic by which a clear response change could be measured - but, as illustrated in Fig.4, shorter stimulus periods gave rise to smaller effects, and as Fig.11b shows, repeated stimulation could be additive. We have opted for a simple stimulus paradigm, widely used, with standard statistical tests available to analyse the results. However, we do not suggest that the effect we have observed is the process used by the cat brain in vivo to analyse the visual world - the real effect may operate at very much shorter time scales, perhaps dealing only in the likelihood of a given set of spikes to be followed by another spike or spikes as the visual scene changes or even as the visual response to the scene develops. There are important recent data which show a decisive role for corticothalamic projections in organizing long-range synchrony and the spatiotemporal patterns of activity generated in the thalamus (Sillito et al., 1994; Contreras et al., 1996), indeed, this may well account for the fact that the effectiveness of electrical stimulation of the optic chiasm was greatly enhanced when paired with an incoming visual (physiological) input, which is much more likely to engage the corticofugal loop (Cudeiro \& Sillito, 1996). There is now also strong evidence for augmentation in the visual system operating at this low level (Ferster \& Lindstrom, 1985; Steriade \& Timofeev, 1997; Timofeev \& Steriade, 1997; Steriade et al., 1998), whereby electrical stimulation can result in increased likelihood of firing after the stimulation has ceased. These latest studies, however, rarely involve in vivo visual responses, and it is important to place these in the context of our own, by 
which we may see in fact a spectrum of response control in time ranging from additional single spikes to large numbers dependent upon the context of the visual stimulus, but, crucially, both spatial and temporal in nature.

\section{Acknowledgements}

This work was supported by the XUGA-13401B96, Vicerrectorado de Investigacion (A Coruña and FIS-97/0402 (Spain).

\section{Abbreviations}

LGN, dorsal lateral geniculate nucleus; LTP, long-term potentiation; NMDA, N-methyl-D-aspartate; PSTH, post-stimulus time histogram; STP, short-term potentiation.

\section{References}

Artola, A.\& Singer, W.(1987) Long term potentiation and NMDA receptors in rat visual cortex. Nature, 330, 649652

Bliss, T.V.P. \& Collingridge, G.L. (1993) A synaptic model of memory: long term potentiation in the hippocampus. Nature, 361, 31-39.

Bliss, T.V.P. \& Gardner-Medwin, A.R. (1973) Long-lasting potentiation of synaptic transmission in the dentate area of the unanaesthetized rabbit following stimulation of the perforant path. J. Physiol. (Lond.), 232, 357-374.

Bliss, T.V.P. \& Lomo, T. (1973) Long-lasting potentiation of synaptic transmission in the dentate area of the anaesthetized rabbit following stimulation of the perforant path. J. Physiol. (Lond.),232, 331-356.

Contreras, D., Destexhe, A., Sejnowski, T.J. \& Steriade, M. (1996) Control of spatiotemporal coherence of a thalamic oscillation by corticofugal feedback. Science, 274, 771-774.

Cox, C.L., Zhou, Q. \& Sherman, S.M. (1998) Glutamate locally activates dendritic outputs of thalamic interneurons. Nature, 394, 478-482.

Cudeiro, J., Rivadulla, C., Rodriguez, R., Grieve, K.L., Martinez-Conde, S. \& Acuña, C.(1997) Actions of compounds manipulating the nitric oxide system in the cat primary visual cortex. J. Physiol. (Lond.), 504, 467478

Cudeiro, J.\& Sillito, A.M.(1996) Spatial frequency tuning of orientation-discontinuity-sensitive corticofugal feedback to the cat lateral geniculate nucleus. J. Physiol. (Lond.), 490, 481-492.

DeAngelis, G.C., Robson, J.G., Ohzawa, I. \& Freeman, R.D. (1992) Organisation of suppression in receptive fields of neurons in cat visual cortex. J. Neurophysiol., 68, 144-163.

Ferster, D.\& Lindstrom, S.(1985) Augmenting responses evoked in area 17 of the cat by intracortical axon collaterals of cortico-geniculate cells. J. Physiol. (Lond.), 367, 217-232.

Fox, K.(1995) The critical period for long-term potentiation in primary sensory cortex. Neuron, 15 , 485-488.

Gilbert, C.D. \& Wiesel, T.N. (1991) The influence of contextual stimuli on the orientation selectivity of cells in the primary visual cortex of the cat. Vision Res., 30, 1689-1701.

Godwin, D.W., Van Horn, S.C., Eriir, A., Sesma, M., Romano, C. \& Sherman, S.M. (1996a) Ultrastructural localization suggests that retinal and cortical inputs access different metabotropic glutamate receptors in the lateral geniculate nucleus. J. Neurosci., 16, 8181-8192.

Godwin, D.W., Vaughan, J.W. \& Sherman, S.M. (1996b) Metabotropic glutamate receptors switch visual response mode of lateral geniculate nucleus cells from burst to tonic. J. Neurophysiol., 76, 1800-1816.

Guido, W., Lu, S.-M., Vaughn, J.W., Godwin, D.W. \& Sherman, S.M. (1995) Receiver operating characteristics (ROC) analysis of neurons in the cat's lateral geniculate nucleus during tonic and burst response modes. Visual Neurosci., 12, 723-741.

Kirkwood, A.\& Bear, M.F.(1994) Hebbian synapses in visual cortex. J. Neurosci., 14, 1634-1645.

Levitt, J.B. \& Lund, J.S. (1997) Contrast dependence of contextual effects in primate visual cortex. Nature, 387, 7376.

Logothetis, N.K., Leopold, D.A. \& Sheinberg, D.L. (1996) What is rivaling during binocular rivalry? Nature, 380, 621-624.

Maffei, L.\& Fiorentini, A.(1976) The unresponsive regions of visual cortical receptive fields. Vision Res., 16, 11311139.

McCormick, D.\& von Krosigk, M.(1992) Corticothalamic activation modulates thalamic firing through glutamate metabotropic receptors. Proc. Natl Acad. Sci. USA, 89, 2774-2778.

Murphy, P.C. \& Sillito, A.M. (1987) Corticofugal feedback influences the generation of the length tuning in the visual pathway. Nature, 329,727-729.

Nelson, J.I. \& Frost, B.J. (1978) Orientation-selective inhibition from beyond the classical receptive visual field. Brain Res., 139, 1689-1701.

O'Connor, J.J., Rowan, M.J. \& Anwyl, R. (1994) Long-lasting enhancement of NMDA mediated synaptic transmission by metabotropic glutamate receptor activation. Nature, 367, 557-559. 
Pape, H.C. \& Eysel, U.T. (1986) Binocular interactions in the lateral geniculate nucleus of the cat: GABAergic inhibition reduced by dominant eye activity. Exp. Brain Res., 61, 265-271.

Reinagel, P., Godwin, D., Sherman, S.M. \& Koch, C. (1999) Encoding of visual information by LGN bursts. J. Neurophysiol., 81, 2558-2569.

Rivadulla, C., Grieve, K.L. \& Cudeiro, J. (1998) Enhanced visual responses in cat dLGN - potentiation by priming with excitatory amino acids. Neuroreport, 9, 653-657.

Sherman, S.M. (1996) Dual response modes in lateral geniculate neurons: mechanisms and functions. Vis. Neurosci., $13,205-213$

Sherman, S.M. \& Guillery, R.W. (1998) On the actions that one nerve cell can have on another: distinguishing "drivers" from "modulators". Proc. Natl Acad. Sci. USA, 95 , 7121-7126.

Sillito, A.M., Grieve, K.L., Jones, H.E., Cudeiro, J. \& Davis, J. (1995) Visual cortical mechanisms detecting focal orientation discontinuities. Nature, 378, 492-496.

Sillito, A.M., Jones, H.E., Gerstein, G.L. \& West, D.C. (1994) Feature-linked synchronization of thalamic relay cell firing induced by feedback from the visual cortex. Nature, 369, 479-482.

Sillito, A.M. \& Kemp, J.A. (1983) The influence of GABAergic inhibitory processes on the receptive field structure of $\mathrm{X}$ and $\mathrm{Y}$ cells in cat dorsal lateral geniculate nucleus (dLGN). Brain Res., 277, 63-77.

Steriade, M.\& Timofeev, I.(1997) Short-term plasticity during intrathalamic augmenting responses in decorticated cats. J. Neurosci., 17, 3778-3795.

Steriade, M., Timofeev, I., Grenier, F. \& Durmuller, N. (1998) Role of the thalamic and cortical neurons in augmenting responses and self-sustained activity: dual intracellular recordings in vivo. J. Neurosci., 18, 64256443.

Timofeev, I.\& Steriade, M.(1997) Intrathalamic mechanisms underlying incremental responses in reticular and relay neurons. J. Neurophysiol., 79, 2716-2729.

Uhlrich, D.J., Tamamaki, N., Murphy, P.C. \& Sherman, S.M. (1995) Effects of brain stem parabrachial activation on receptive field properties of cells in the cat's lateral geniculate nucleus. J. Neurophysiol., 73, 2428-2447.

Vidyasagar, T.R. (1984) Contribution of inhibitory mechanisms to the orientation sensitivity of cat dLGN neurones. Exp. Brain Res., 55, 192-195 